\title{
Tourette's disorder and other tic disorders in DSM-5: a comment
}

\author{
Veit Roessner $\cdot$ Pieter J. Hoekstra • \\ Aribert Rothenberger
}

Received: 25 September 2010/Accepted: 23 October 2010/Published online: 13 November 2010

(C) The Author(s) 2010. This article is published with open access at Springerlink.com

\begin{abstract}
Classification of tic disorders will be revised in the forthcoming edition of the Diagnostic and Statistical Manual of Mental Disorders (5th ed.; DSM-5). We do not support the suggestion to move tic disorders to "Anxiety and Obsessive-Compulsive Disorders", if the section "Disorders Usually First Diagnosed in Infancy, Childhood, or Adolescence" is not retained. Other than that, most proposed changes of the criteria for tic disorders contain a number of welcome improvements, e.g., the more unified definition of tics including the removal of the term "stereotyped" and the better capture of the temporal pattern of tics (e.g., removal of the maximum 3 months criterion for a tic-free period in chronic tic disorders). But, unfortunately there are some inconsistencies in detail, e.g., the unification of diagnostic criteria for tic disorders had not been consistently pursued in transient tic disorder. In sum, the proposed DSM-5 criteria could be seen as an important step forward particularly in clinical routine. However, continued research is needed to justify the existing and proposed classification of tic disorders as well as to better clarify what other changes should be made in the DSM- 5 and beyond.
\end{abstract}

Keywords DSM-5 - Tic disorders - Tourette syndrome

V. Roessner $(\bowtie)$

Department of Child and Adolescent Psychiatry, University

of Dresden, Goetheallee 12, 01309 Dresden, Germany

e-mail: veit.roessner@uniklinikum-dresden.de

P. J. Hoekstra

Department of Child and Adolescent Psychiatry,

University Medical Center Groningen, University of Groningen,

Hanzeplein 1, 9713 GZ Groningen, The Netherlands

A. Rothenberger

Department of Child and Adolescent Psychiatry, University of Göttingen, Von-Siebold-Straße 5, 37075 Göttingen, Germany

\section{Introduction}

Tic disorders have higher prevalence rates than previously thought, of up to 3-4\%. For Tourette's disorder (TD; also known as Gilles de la Tourette syndrome or Tourette syndrome), a prevalence of $1 \%$ (range $0.05-3 \%$ ) was given [8]. Typically, first tics occur in childhood and reach their peak severity early in the second decade of life often followed by a time of remission of tics and tic-associated impairment $[4,15]$. Generally, tic disorders have a favorable prognosis: follow-up studies of tic disorders suggest that approximately one-third of children with tic disorders are essentially symptom free as adults; another third will have mild symptom severity that does not require clinical attention. Only $<20 \%$ of persons with TD continue to experience a moderate level of impairment of global functioning by the age of 20 years [3]. Adults who still have symptoms severe enough to come to clinical attention are therefore unusual representatives of all subjects who have received a diagnosis of tic disorders. Coexisting conditions are rather the rule than the exception. Although there seems to be a clear and unique defining symptomatology of tics, the inter- and intraindividual variability is a challenge to classification.

\section{General comments on tic disorders in the proposed DSM-5}

The current edition of the Diagnostic and Statistical Manual of Mental Disorders (DSM-IV-TR) defines tics as sudden, rapid, recurrent, nonrhythmic, stereotyped motor movements or vocalizations. It is very welcome that in the proposed DSM-5 the definition of a tic would be made consistent for all the tic disorders. Additionally, the term 
"stereotyped" would be removed from the definition of tics to avoid confusions in distinguishing between tics and stereotypic movements. However, in their "Comment on the proposed changes to the diagnostic criteria and classification of Tourette's disorder in the forthcoming Diagnostic and Statistical Manual-V (DSM-5)" the American Tourette Syndrome Association (TSA) expresses its hope that the DSM-5 committee will consider revising the definition of a tic so that it is both accurate and does not lead to misdiagnoses [16]. In our opinion, this is all the more needed as clear diagnostic criteria for tics as well as studies on this issue have been few [7].

The phenomenological overlap with the commonly co-occurring psychiatric conditions attention deficit/hyperactivity disorder (ADHD) and obsessive-compulsive disorder (OCD) could be a challenge in some cases [5]. In clinical samples of TD, about half of the cases also meet criteria for ADHD and vice versa TD is seen in about $20 \%$ of children with ADHD [14, 15]. Besides, patients with TD also suffer frequently from obsessive-compulsive symptoms or OCD (about 50\%). Especially, the need to achieve a "just right" feeling in tic disorders can be seen as an indicator for a continuum between tic disorders and OCD [12].

However, given the high rate of tic remission before the typical onset of OCD or OCD-like behavior [6] and the high rate of co-occurrence with $\mathrm{ADHD}$, one recommendation of the DSM-5 working group has to be questioned. They propose that tic disorders should be moved to "Anxiety and Obsessive-Compulsive Disorders", if the section "Disorders Usually First Diagnosed in Infancy, Childhood, or Adolescence" is not retained. One possible reason for this suggestion might be the fact that there are virtually no data on familiality of ADHD and tic disorders particularly from the ADHD perspective, in contrast to tic disorders and early onset OCD. Therefore, we fully agree with the TSA's "strong recommendation" [16] that in case of removal of the category of "Disorders Usually First Diagnosed In infancy, Childhood or Adolescence" tic disorders should be placed under a different or newly created category that is more suitable for these neuropsychiatric disorders. Additionally, the TSA suspects that the enormous worldwide effort "to dispel the misleading notion that TD is, at its root, a psychiatric condition" will be undone.

Already DSM-IV-TR had dropped the DSM-4 requirement of tics to cause clinically significant distress or impairment and this is retained in DSM-5. Particularly in tic disorders, such an "impairment criterion" would have hampered genetic research because in DSM-4 when a child whose presentation clearly meets the tic symptomatology criteria for TD, but who does not have significant impairment or distress from the tics, no diagnosis of a tic disorder could be made. Moreover, recent studies have shown that it is often not the tics but comorbid conditions such as ADHD that are associated with social and academic impairment [1, 9-11].

\section{Comments on proposed changes of criteria for existing diagnoses of tic disorders in DSM-5}

The DSM-IV-TR distinguishes several forms of tic disorders. The two chronic tic disorders, chronic motor or vocal tic disorder and TD require the presence of tics at some time during the illness, with onset before age 18 years, throughout a period of more than 1 year, and without a tic-free period exceeding three consecutive months during this period. Moreover, tics should not be due to the direct physiological effects of a substance (e.g., stimulants) or a general medical condition (e.g., Huntington's disease or postviral encephalitis). In chronic motor or vocal tic disorder tics should be limited to either motor or vocal tics, whereas in TD both multiple motor and one or more vocal tics have to be present.

Apart from these two chronic tic disorders, DSM-IV-TR contains criteria for transient tic disorder, requiring tics to be present for at least 4 weeks, but for no longer than 12 consecutive months. Although the differentiation between transient and chronic tic disorders has proven its clinical usefulness, the exact time criteria, i.e., tics are present shorter or longer than 1 year as well as the maximum 3-month tic-free interval given for chronic tic disorders, have never been supported by valid data such as a longitudinal study on tics in an epidemiological sample.

Nevertheless, in accordance with the TSA [16], we welcome that in DSM-5 a diagnosis of chronic tic disorders can be made if tics persist for more than 1 year, beginning at first onset and regardless of a tic-free period during that time. In our experience, the retrospective differentiation of tic-free intervals is a difficult matter in some cases and most clinicians make a diagnosis of a chronic tic disorder based on the duration of symptoms from first tic onset and do not adhere to the maximum 3-month tic-free interval. Hence, removal of the 3 months criterion is consistent with clinical practice and simplifies diagnosing chronic tic disorders. In this context it has to be stated that we have almost no data on phenomenology, prognostic factors, etiology, and pathophysiology of transient tic disorders possibly revealing differences to chronic tic disorders. More data in this regard would also be most welcome for clinicians. In terms of the classificatory differentiation between chronic motor or vocal tic disorder versus TD, similar problems due to the lack of specific studies looking for differences between both diagnoses have to be mentioned. Such a differentiation seems to be of no clinical relevance aside from the fact that the label TD even for the 
combination of chronic but very mild motor and vocal tics alarms several parents more than necessary.

In addition, the unification of diagnostic criteria for tic disorders had not been consistently pursued in transient tic disorder. That is, only DSM-IV-TR criteria for the chronic tic disorders, and not those for transient tic disorder, state that tics should not be due to the direct physiological effects of a substance (e.g., stimulants) or a general medical condition (e.g., Huntington's disease or postviral encephalitis). Moreover, the DSM-IV-TR criteria for chronic tic disorders, explicitly point to the waxing and waning nature of tics by stating that tics usually occur in bouts nearly every day or intermittently, whereas this is not present in the criteria for transient tic disorder.

In DSM-5 it is proposed to change the name "Transient Tic Disorder" to "Provisional Tic Disorder" due to problems with the 1 year criterion that can only be fulfilled by a patient in retrospective. When a child would have tics since say 8 months it is at that moment unknown whether this will turn out to be a transient or chronic tic disorder. Only time can tell in such a case. The TSA and we support the proposed revision, although in terms of clinical care the term "provisional" may mitigate the high severity of the child's tics urgently requiring sufficient treatment. Nevertheless, from a research perspective the retrospective differentiation between provisional (still unclear how long tics will be present) and transient tics (definitely a presence of tics $<1$ year) would make sense. Surprisingly, the specification "Single Episode" or "Recurrent" mentioned in DSM-IV-TR was removed in the proposed DSM-5 criteria; a rationale is not provided.

Finally, DSM-IV-TR included a rest category, "Tic disorder Not Otherwise Specified", for disorders characterized by tics that do not meet criteria for a specific tic disorder. Examples include tics lasting $<4$ weeks or tics with an onset after age 18 years. In DSM-5 the proposed removal of the 4 weeks criterion will be an important step forward, because "the number of cases of Tic Disorders Not Otherwise Specified should be fewer". The same has to be stated concerning the proposed removal of the stimulants in DSM-5 as an example of tic induction due to the direct physiological effects of a substance. We welcome the removal because recent evidence neither points to a relationship between stimulants and onset [13] nor exacerbation of existing tics [2].

\section{Comments on proposed addition of diagnoses of tic disorders in DSM-5}

While in line with the general structure of DSM, the introduction of a new category "Substance-Induced Tic

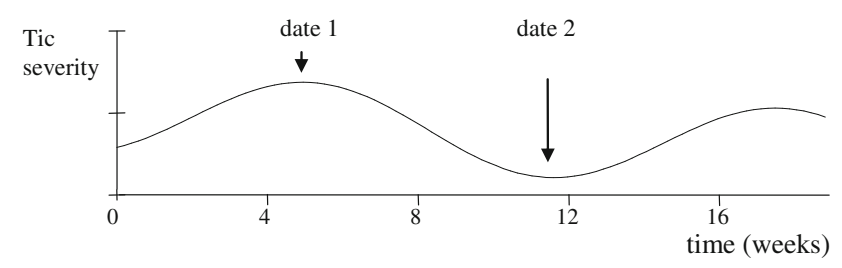

Fig. 1 Evaluation of treatment effects in TS in light of natural waxing and waning. At date 1 a therapeutic intervention could be followed by tic reduction despite ineffectiveness or even a potential to actually increase tics. Accordingly, such a reduction cannot readily be ascribed to causal mechanisms of the intervention; the natural waxing and waning of the tics must be considered. Correspondingly, a therapeutic intervention at date 2 could be followed by an increase of TS symptomatology despite its potential to reduce tics. The therapeutic intervention might attenuate the natural waxing of the tics. Conclusion: Meaningful appraisal of treatment efficacy in TS can only be given in most cases after longer time

Disorder" in DSM-5 has only limited clinical utility. Reports about an assumed causal relationship between medication or drug use (DSM-5 mentions "e.g., cocaine, methamphetamine") and first onset of tics are very scarce and have to be questioned in view of the waxing and waning of tics that is an obstacle for causal conclusions based on single case reports or case series (see Fig. 1). We would suggest to subsume these rare cases under the label "Tic Disorder Not Otherwise Specified". Independently of their categorization here again, more knowledge about etiology and pathophysiology of tics including their severity fluctuations would be helpful particularly for prognostic and therapeutic issues.

The same has to be stated concerning the base of data on which a new diagnosis "Tic Disorder Due to a General Medical Condition" is to be introduced (proposed DSM-5 criteria mention “e.g., stroke, Huntington's disease, postviral encephalitis").

\section{Concluding remarks}

In summary, the proposed DSM-5 criteria for tic disorders contain a number of welcome improvements, in terms of more unified definition of tics, better capture of the temporal pattern of tics, and renaming of "Transient Tic Disorder" to "Provisional Tic Disorder". However, in our view there is broad consensus that the section "Disorders Usually First Diagnosed In infancy, Childhood or Adolescence" is the best category for tic disorders and in case of removal of that section tic disorders should not be included in a section entitled "Anxiety and ObsessiveCompulsive Disorders". Also, the proposed new categories "Substance-Induced Tic Disorder" and "Tic Disorder Due to a General Medical Condition" have very limited clinical 
utility; in general, we favor descriptive criteria over criteria that require a causal relationship, which may be hard to demonstrate.

Additionally, several gaps of knowledge in the field of tic disorders should be overcome by further investigations before some of the changes in the classificatory system of tic disorders seem necessary or justified. Diagnosing as well as symptom measurement need to be put on a sounder basis of evidence by studies comparing different diagnostic algorithms and questionnaires. Closely related, subtyping of tic disorders according to specific aspects e.g., based on the presence of commonly co-occurring disorders such as ADHD or OCD should be approached with increasing impetus.

Open Access This article is distributed under the terms of the Creative Commons Attribution Noncommercial License which permits any noncommercial use, distribution, and reproduction in any medium, provided the original author(s) and source are credited.

\section{References}

1. Banaschewski T, Neale BM, Rothenberger A, Roessner V (2007) Comorbidity of tic disorders \& ADHD: conceptual and methodological considerations. Eur Child Adolesc Psychiatry 16(Suppl 1):5-14

2. Bloch MH, Panza KE, Landeros-Weisenberger A, Leckman JF (2009) Meta-analysis: treatment of attention-deficit/hyperactivity disorder in children with comorbid tic disorders. J Am Acad Child Adolesc Psychiatry 48:884-893

3. Bloch MH, Peterson BS, Scahill L, Otka J, Katsovich L, Zhang H, Leckman JF (2006) Adulthood outcome of tic and obsessivecompulsive symptom severity in children with Tourette syndrome. Arch Pediatr Adolesc Med 160:65-69

4. Coffey BJ, Biederman J, Geller D, Frazier J, Spencer T, Doyle R, Gianini L, Small A, Frisone DF, Magovcevic M, Stein N, Faraone SV (2004) Reexamining tic persistence and tic-associated impairment in Tourette's disorder: findings from a naturalistic follow-up study. J Nerv Ment Dis 192:776-780
5. Freeman RD (2007) Tic disorders and ADHD: answers from a world-wide clinical dataset on Tourette syndrome. Eur Child Adolesc Psychiatry 16(Suppl 1):15-23

6. Leckman JF (2002) Tourette's syndrome. Lancet 360:1577-1586

7. Paszek J, Pollok B, Biermann-Ruben K, Muller-Vahl K, Roessner V, Thomalla G, Robertson MM, Orth M, Schnitzler A, Munchau A (2010) Is it a tic?-Twenty seconds to make a diagnosis. Mov Disord 25:1106-1108

8. Robertson MM, Eapen V, Cavanna AE (2009) The international prevalence, epidemiology, and clinical phenomenology of Tourette syndrome: a cross-cultural perspective. J Psychosom Res $67: 475-483$

9. Roessner V, Banaschewski T, Fillmer-Otte A, Becker A, Albrecht B, Uebel H, Sergeant J, Tannock R, Rothenberger A (2008) Color perception deficits in co-existing attention-deficit/ hyperactivity disorder and chronic tic disorders. J Neural Transm 115:235-239

10. Roessner V, Becker A, Banaschewski T, Rothenberger A (2007) Executive functions in children with chronic tic disorders with/ without ADHD: new insights. Eur Child Adolesc Psychiatry 16(Suppl 1):36-44

11. Roessner V, Becker A, Banaschewski T, Rothenberger A (2007) Psychopathological profile in children with chronic tic disorder and co-existing ADHD: additive effects. J Abnorm Child Psychol 35:79-85

12. Roessner V, Becker A, Banaschewski T, Rothenberger A (2005) Tic disorders and obsessive compulsive disorder: where is the link? J Neural Transm Suppl 69-99

13. Roessner V, Robatzek M, Knapp G, Banaschewski T, Rothenberger A (2006) First-onset tics in patients with attention-deficithyperactivity disorder: impact of stimulants. Dev Med Child Neurol 48:616-621

14. Rothenberger A, Roessner V, Banaschewski T, Leckman JF (2007) Co-existence of tic disorders and attention-deficit/hyperactivity disorder-recent advances in understanding and treatment. Eur Child Adolesc Psychiatry 16(Suppl 1):1-4

15. Schlander M, Schwarz O, Rothenberger A, Roessner V (2010) Tic disorders: administrative prevalence and co-occurrence with attention-deficit/hyperactivity disorder in a German community sample. Eur Psychiatry (epub ahead of print)

16. Tourette Syndrome Association (TSA) (2010) Comment on the proposed changes to the diagnostic criteria and classification of Tourette's disorder in the forthcoming Diagnostic and Statistical Manual-V (DSM-5) 